\title{
SEISMOLOGY OF SOUTHERN BE STARS
}

\section{EDUARDO JANOT-PACHECO and NELSON VANI LEISTER \\ Depto. Astronomia, IAG-USP, Brazil}

\section{The survey: search for rapid variability in Be stars}

We have started in 1990 a search for moving bumps in the HeI $\lambda 667.8$ $\mathrm{nm}$ of mainly southern, bright Be stars. The objects of our sample have been selected on the basis of photometric variability (Cuypers et al., 1989). High resolution $(R \geq 30,000)$, high signal-to-noise ratio $(S / R \geq 300)$ spectroscopic observations have been performed at the brazilian Laboratório Nacional de Astrofísica with a CCD camera attached to the coudé spectrograph of the $1.60 \mathrm{~m}$ telescope (e.g. Table I). Several hundred spectra have been taken during the last three years. Photometric observations simultaneous with spectroscopy were made on the same site in July 1992 with a two-channel photometer (Stromgren $b$ filter) and a CCD camera (Johnson $B$ filter) installed at two $0.60 \mathrm{~m}$ telescopes. The idea is try to disentangle the controversy between NRP and RM models with the help of simultaneous spectroscopy and photometry.

TABLE I

Some Be stars observed at L.N.A.

\begin{tabular}{llll}
\hline Star & Epoch & Type of Observation & Remarks \\
\hline \multirow{2}{*}{$\alpha$ Eri } & $1990-93$ & Spectroscopy & $l=|m| \sim 10$ \\
& $1990-93$ & Photometry & \\
& 1993 & CCD & \\
$\lambda$ Eri & 1990 & Spectroscopy & bumps? \\
& 1993 & Photometry & \\
& 1993 & CCD & \\
$\eta$ Cen & $1990-93$ & Spectroscopy & $l=|m| \sim 14$ \\
$\zeta$ Oph & $1990-93$ & Spectroscopy & $l=|m| \sim 7$ \\
\hline
\end{tabular}

\section{Some results}

Moving subfeatures are seen in $\sim 50 \%$ of the stars in our sample. $\alpha$ Eri is discussed elsewhere in this meeting (Leister et al., 1993a this volume). 
- $\eta$ Cen (B2 IVe)-Subfeatures ( $<1 \%$ of the continuum were detected moving from blue to red through the line profiles. A detailed analysis of the data is presented elsewhere (Leister et al. 1993b). The line profile variations have been examined in terms of nonradial oscillations: from the acceleration of the bumps at the line center and the average time delay between subfeatures crossings. We found $l=|m|=14 \pm 4$, the error being essentially due to the $10 \%$ formal error in Vsin $i$ (Slettebak 1982). Data are also compatible with spheroidal tesseral NRP modes with $l \cong 7, m \cong 6$ (see Kambe and Osaki 1988). The travelling period of bumps is greater than the stellar rotation period, showing that NRP are retrograde in the corotating frame. Bumps can be followed over a range of at least $\pm 400 \mathrm{kms}^{-1}$ which is larger than $V \sin i$ of the star. The azimuthal NRP degree remained unchanged during the time span of our observations. This standing character of the overall stellar pattern from 1990 to 1992 shows that the (presumed) NRP phenomenon in $\eta$ Cen can be stable during at least two years. This result must be taken into account in future theoretical work.

- $\zeta$ Oph (O9.4 Vn)- The analysis of observational data on this object followed the same script as for $\eta$ Cen (see above). Bumps are quite preeminent in this object. Preliminary results were presented by Janot-Pacheco et al. (1991). A quick look on the velocity residual for 1990 suggests $l=|m| \cong$ $7 \pm 2$.

This campaign on analysis of rapid variability among Be stars is jointly conducted by Departamento de Astronomia, IAG/USP (Brazil), and by DASGAL Observatoire de Paris-Meudon (France)

\section{Acknowledgements}

This research is supported in part by FAPESP and CNPq (Brazil), and by CNRS (France).

\section{References}

Cuypers J., Balona L.A. and Marang F.: 1989, $A \& A$ 81, 151

Janot-Pacheco E., Leister N.V., Quast G.R., Torres C.A.P.C.O.,: 1991, Rapid Variability of OB Stars: Nature and Diagnostic Value, ESO workshop, ed. D. Baade , 45

Kambe E.,Osaki Y.: 1988, PASP 40, 313

Leister N.V., Janot-Pacheco E., Buck M.T.C., Dias M.P., Hubert A.M., Hubert H., Floquet $M$. and Briot D.: 1993a, this volume,

Leister N.V., Janot-Pacheco E., Hubert A.M., Hubert H., Floquet M. and Briot D.: 1993b, A\& $A$ submited ,

Slettebak A.: 1982, ApJS 50, 55 